\title{
Mood based Music System
}

\author{
A. S. Mali ${ }^{1 *}$, A. A. Kenjale ${ }^{2}$, P. M. Ghatage ${ }^{3}$, A. G. Deshpande ${ }^{4}$ \\ ${ }^{1}$ Dept. of CSE, Rajarambapu Institute of Technology, Shivaji University, Sakharale, India \\ ${ }^{2,3,4}$ Computer Science and Engineering, Rajarambapu Institute of Technology, Islampur, India
}

Available online at: www.isroset.org

Received: 11/May/2018, Revised: 23/May/2018, Accepted: 14/Jun/2018, Online: 30/Jun/ 2018

\begin{abstract}
Music is a significant element of life. People take its help to evoke their emotions and prefer listening to songs according to their mood. It takes a lot of efforts to find appropriate music from the list for the particular emotional state. Music players in today's world are not giving priority to the emotional state of a person. The aim of this paper is to develop music system which considers human emotions into account. The emotional state can be interpreted from facial expressions through the webcam. We have utilized the CNN classifier to build a neural network model. This model is trained and subjected to detect mood from facial expressions using OpenCV. Songs belonging to particular sentiments are classified on the basis of tempo feature in beats per minute. A system generates music playlist based on that detected mood. Our music player will play that generated music playlist to improves user's mood.
\end{abstract}

Keywords - Machine learning, Image processing, PyQt5, OpenCV, Convolutional Neural Network (CNN), Haar Cascade, Pygame

\section{INTRODUCTION}

Music is an essential component of our daily life. We listen to songs as per our mood. Music is one of the media of entertainment and even imparts a therapeutic approach. It is important to play an appropriate song on the particular emotional state. Existing music player satisfies the user's basic requirements, yet the user has to face the task of manually browsing through the playlist of songs and select songs based on his current mood and behavior.

This project is based on the principle of detection of human emotions to play appropriate songs for current emotional state. The current emotional state of human being can be easily observed through their facial expressions. It can be achieved with help of image processing and machine learning techniques. In music terms, tempo is speed of pace of given piece, usually measured in BPM (Beats Per Minute). Songs with same sentiments can be detected using tempo feature. By extracting those features songs can be categorized into different mood based categories [1].

Our project aims to recommend and play the appropriate music, based on user's current emotional state with the help of image processing techniques through user's facial expressions

\section{RELATED WORK}

Sentiment-Based Music Play System [1], has implemented RASPBERRY-PI based module for mood detection and generate music playlist. To capture the voices and conversations in the room, a microphone is connected to Raspberry Pi. They have converted that speeches into text for speech-to-text conversion. They have used the Python library for Speech Recognition 3.1.3. The generated text is passed through naïve Bayesian classifier for the sentimental analysis of the text. They have used the tempo of the song as the attribute to be related to the sentiment in the room. For example, BPM values for the mood filled with happiness, anger, and sorrow will be 125, 130, 85 BPM respectively. The system will play a song on the basis of sentimental analysis of text generated from speeches in the room.

There is ample work has been done in the field of face based emotion detection. Proposed mood based music player[2], it scans memory for audio files, and classifies audio files using audio extraction module. After dividing audio files into mood based segregation, it captures image from device camera. Feature detection is done with the help of Viola and jones algorithm [3]. With help of OpenCV libraries, it recognizes the emotional state and device plays music accordingly the mood.

In[4], they have proposed a mood detection system. They have used modern CNN for building framework. Their architecture is fully-convolutional neural network that contains 4 residual separable convolutions where each convolution is followed by a batch normalization operation and ReLU activation. Architecture has 60000 parameters. which is corresponds to reduction of 10x of Naive and $80 \mathrm{X}$ of original CNN. They have achieved $66 \%$ of accuracy in mood detection. 
In[8], they have proposed a bimodal emotion recognition system with the combination of facial expressions and speech signals. The models obtained from a bimodal corpus with six acted emotions and ten subjects were trained and tested with different classifiers, such as Support Vector Machine, Naive Bayes and K-Nearest Neighbor. It's result reveals that facial expression gives better performance compared to speech and combination of both also gives better performance with SVM classifier.

\section{METHODOLOGY}

The mood-based music system is computer-based software that focuses on implementing mood detection. It is a prototype of a new product that merges some separate interfaces: face detection, facial expression recognition, Playlist generation, play music.

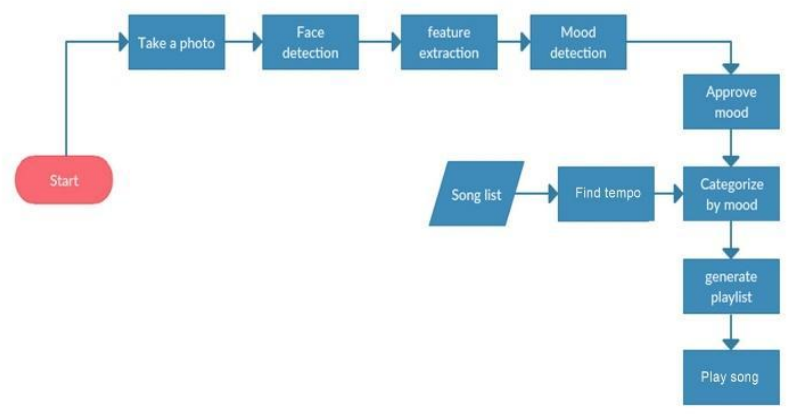

Figure 1: Workflow diagram

\section{- Face detection}

System will take real time input from web camera. We have used OpenCV libraries to implement face detection through Haar cascade classifier[5]. Haar cascade classifier implemented in stages by application of features grouped into criteria such that it will discard the unwanted part from further analysis. Face detection algorithm detects face and further it will extract required features from it.

\section{- Mood detection}

Different muscles can predict human emotional state. We have used CNN classifier to detect emotional state. CNN is made up of neurons with learnable weights and biases. it consist of input and output layers. Each neuron receives several inputs, and pass it through convolution operation function to create an output layer. This function emulates the response of an individual neuron to visual stimuli. The it takes a weighted sum over all inputs. Each convolutional neuron processes data only for its receptive field.So a fully connected neural network made up of number of input layers, that can be used to learn features and differentiate data. The model we have used is trained using FER-2013 dataset.[7] This dataset contains 35,887 grayscale images where each image belongs to one of the classes \{"angry", "disgust", "fear", "happy", "sad", "surprise", "neutral"\}. This model achieved an accuracy of $66 \%$ in this dataset.[4]. Model weights are saved to HDF5 format. This format is a grid format that is ideal for storing multi-dimensional arrays of numbers. By importing this model in Python, mood can be decided from detected face. For accurate decision, we took frequent mood in time interval of 30 seconds as final mood. Finalized mood is passed to music player module to recommend appropriate song.

- Playlist generation

By using Echonest API, we can calculate the tempo of song i.e. BPM (beats per minute). According to the tempo values, we have categorized the songs according to the sentimental categories. BPM values for the mood filled with happiness, anger, and sorrow will be 125, 130, 85 BPM respectively. Finalized mood is imported to this playlist generation module, so that it can recommend appropriate playlist on basis of finalized mood.

- Music player

We have designed our music player's GUI using Python PyQt5 package. It is Python binding for Qt5. Music player imports finalized mood and loads recommended playlist from their modules. And plays songs from that generated playlist. To load these sound objects and control playback, Python's Pygame package is used. From Pygame package, Pygame mixer module is used for loading sound objects and controlling them. By using pygame.mixer module, we have given basic functionalities to our music player so that user can pause the playback, resume the playback, playing previous and next song in playlist, increase and decrease system volume.

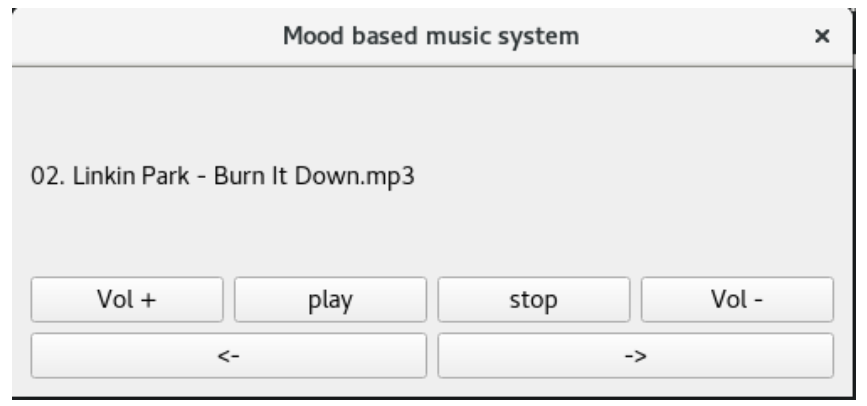

Figure 2: GUI of music player 


\section{RESUlts}

Result of mood based music system can be seen through accuracy in mood detection and in songs recommendation. It is hard to find accurate human emotion only through only one parameter. But with facial expression it can be detected up to some extents. Result of mood detection through facial expression under proper lightning condition can be seen in figure 3. Model we have used has achieved $66 \%$ of accuracy. As it is totally computer based system it understands emotions in the way we trained it. System takes that mood and generates music playlist for that mood accurately. System is able to play most of the songs from recommended playlist.
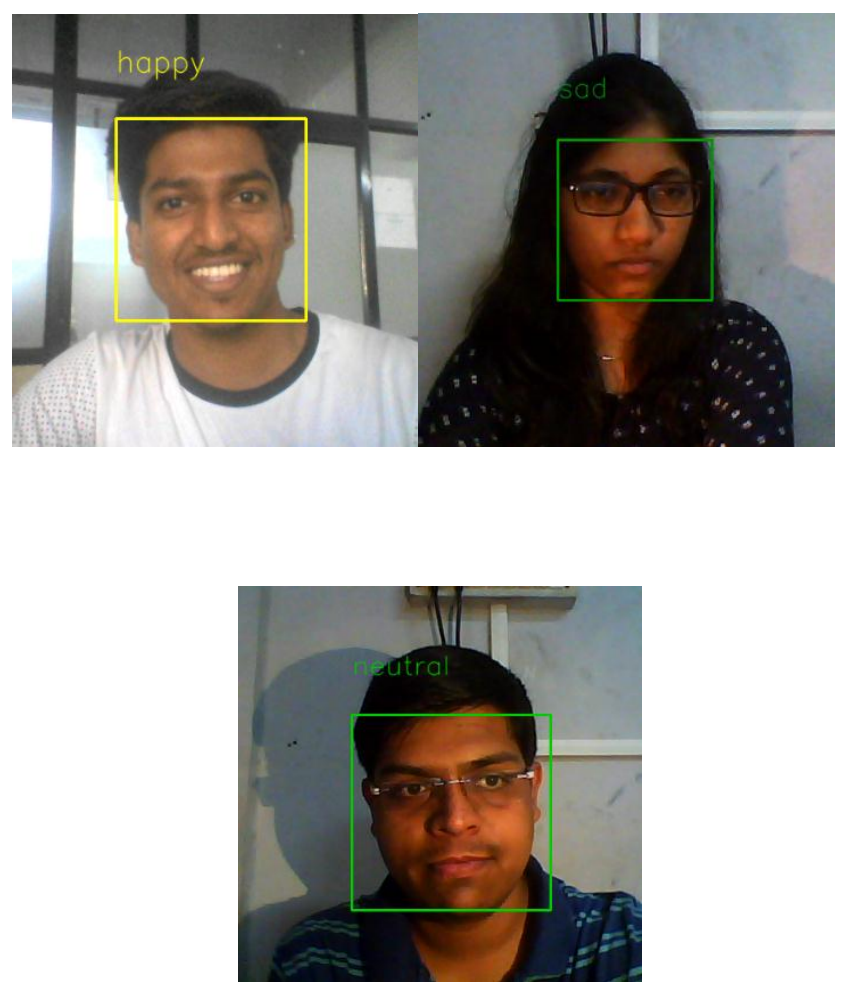

Figure 3: Screenshots of emotion detection

\section{DISCUSSION}

Web camera with better technical specifications gives better result. Proper lightning condition is required. Song choices may vary from person to person.

\section{CONCLUSION and Future Scope}

In this mood-based music system, we have implemented real-time facial emotion recognition using $\mathrm{CNN}$ algorithm in OpenCV. Recognized emotion is passed to music player which has sorted music list according to mood. This music list is sorted by using the tempo of songs. By passing this mood, the system plays appropriate songs in that list to improve user's mood.

This system minimizes the efforts of user to select music according to user's current emotional state. It may reduce physical stress and also act as a boon for the music therapy system. We believe this sentiment-based music system will help user in enjoying their music much more.

In future, this system can be enhanced with capability of detecting mood using voice, facial expressions and body postures to get more accuracy. Also, we can be improved analysis and sorting of user's playlist by mood at run time. At this stage, support of Pygame to .mp3 files is lagging in Linux based operating system. We can be provided more accurate API or Python module which supports all .mp3 files.

\section{Abbreviations and Acronyms}

CNN: Convolutional Neural Network

OpenCV: Open Source Computer Vision Library

\section{ACKNOWLEDGMENT}

We would like to thank our project guide Prof. A. S. Mali sir and Mrs. A. K. Ingale for the valuable support and guidance gave us on every step of the project execution. We would also like to thank the project review committee member Prof. A. M. Jagtap sir. We would also like to express our gratitude to Dr. N. V. Dharwadkar, Head of Department, Department of Computer Science and engineering, Rajarambapu Institute of technology, Rajaramnagar who helped us to accomplish this work.

\section{REFERENCES}

[1] V. Patchava, P. Jain,R. Lomte, P. Shakthi, H. B. Kandala, "Sentiment Based Music Play System"

[2] P. Pathak, Prof. S. Aranjo, "Mood based Music Player Karan Mistry".

[3] Viola, P., \& Jones, M. J., "Robust Real-Time Face Detection", International Journal of Computer Vision, 57(2), 137-154 (2004).

[4] O. Arriaga, G. Paul, M. Valdenegro, "Real-time Convolutional Neural Networks for Emotion and Gender Classification"

[5] Face detection in OpenCV using Haar cascade

[6] The Echo Nest Developer Center.

[7] Kaggle FER2013 open source dataset for face

[8] A. R. Patel, A. Vollal, P. B. Kadam, S. Yadav, R. M. Samant,"MoodyPlayer: A Mood based Music Player".

[9] C. Jenefer, S. Leena, M. Nirmala, Dr. J. SelvaKumar, "A Survey: Expression Based Music Player". 


\section{Authors Profile}

Mr. A.S.Mali pursed M.Tech (CSE) from Rajarambapu Institute of Technology, Rajaramnagar Islampur Dist: Sangli $\mathrm{He}$ is working as Assistant Professor in the Department of Computer Science and Technology at RIT, Islampur. $\mathrm{He}$ has published more than 12 research papers in reputed international journals including IEEE, Elsevier and it's also available

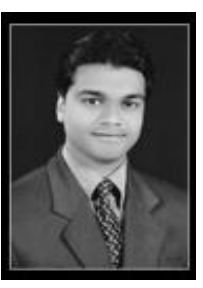
online. His main search work focuses on Internet of Things (IoT), Cloud Computing, Big Data Analytics. He has 6 years of teaching experience

Mr. A. A. Kenjale pursed Bachelor of Technology from Shivaji University in the Department of Computer Science Engineering. $\mathrm{He}$ is currently working as Member of Technical Staff in ProtoTech Solutions.

Ms. P. M. Ghatage pursed Bachelor of Technology from Shivaji University in the Department of Computer Science Engineering. She is selected for the position of Assistant System Engineer-Trainee in Tata Consultancy Services Limited.

Mr. A. G. Deshpande pursed Bachelor of Technology from Shivaji University in the Department of Computer Science Engineering. He is currently working as Technical Support Engineer-Trainee in Redhat.

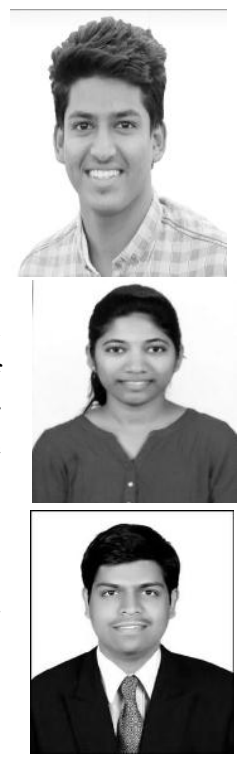

